\title{
Phosphatase inhibitor prevents protein-misfolding diseases
}

The misfolding and aggregation of specific proteins underlies the pathology of several neurodegenerative disorders. Now, writing in Science, Das et al. identify a smallmolecule protein phosphatase 1 inhibitor, sephin 1, that protects cells from protein-misfolding stress, preventing disease development in mouse models of Charcot-MarieTooth 1B (CMT1B) and amyotrophic lateral sclerosis.

The accumulation of abnormally folded proteins in cells causes stress, activating the stress responses to restore proteostasis. As part of the response to many different stresses, eukaryotic translation initiation factor $2 \alpha$ (eIF $2 \alpha)$ is temporarily phosphorylated - a process that inhibits protein translation,

allowing chaperones to catch up on protein folding.

The anti-hypertensive drug guanabenz (GBZ) prolongs eIF2 $\alpha$ phosphorylation by inhibiting PPP1R15A - the regulatory subunit of the stress-induced
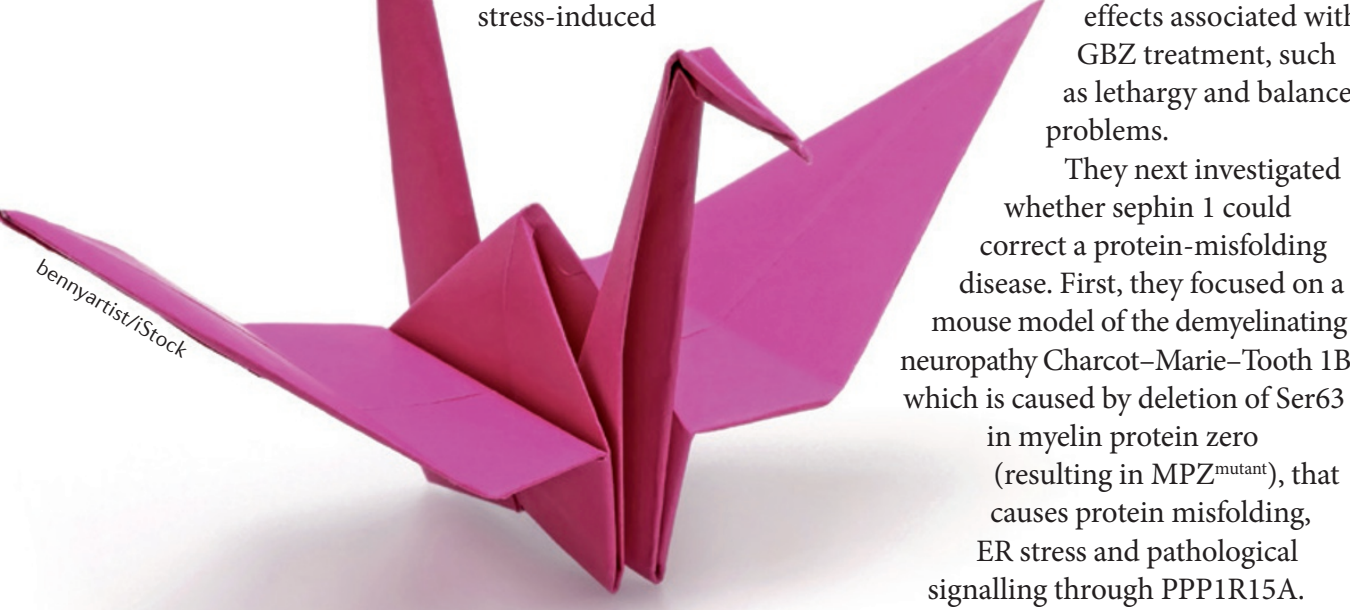

eIF2 $\alpha$ phosphatase PPP1R15A-PP1c, which mediates eIF2 $\alpha$ dephosphorylation. However, although GBZ has been shown to prevent misfolded protein accumulation and slow down disease in mouse models of neurodegenerative disorders, its use is associated with side effects owing to its affinity for the $\alpha 2$-adrenergic receptor. Das and colleagues therefore set out to identify a small-molecule selective inhibitor of PPP1R15A.

To do this, the authors synthesized a panel of GBZ derivatives and selected one missing a chlorine residue, named sephin 1 . In cells exposed to the endoplasmic reticulum (ER) stress inducer tunicamycin, sephin 1 selectively inhibited PPP1R15A, prolonged eIF2 $\alpha$ phosphorylation and delayed translation recovery, thereby protecting cells from cytotoxic ER stress. Importantly, as sephin $1 \mathrm{did}$ not interact with the a 2 -adrenergic receptor, mice treated orally with sephin 1 for one month did not exhibit the adverse effects associated with GBZ treatment, such as lethargy and balance problems.

They next investigated whether sephin 1 could correct a protein-misfolding disease. First, they focused on a inating neuropathy Charcot-Marie-Tooth 1B, in myelin protein zero (resulting in $\mathrm{MPZ}^{\text {mutant}}$ ), that causes protein misfolding, signalling through PPP1R15A.
In dorsal root ganglia cultures from MPZ ${ }^{\text {mutant }}$ mice, 2 -week sephin 1 treatment rescued myelination defects and decreased the expression of ER-stress genes. Furthermore, postnatal treatment of MPZ ${ }^{\text {mutant }}$ mice with sephin 1 orally twice a day on days 28-61 completely prevented motor defects at 4 months, rescued myelin thickness around axons in sciatic nerves and reduced the levels of ER-stress markers, without any obvious adverse effects.

Similarly, in a mouse model of familial amyotrophic lateral sclerosis (FALS) - a motor neuron disease caused by mutant and misfoldingprone superoxide dismutase 1 (SOD1) - sephin 1 treatment once a day from 4 to 11 weeks of age prevented motor deficits, motor neuron loss and progressive weight loss. These beneficial actions occurred in conjunction with prevention of the accumulation of mutant SOD1 protein and decreased ER-stress markers in transgenic spinal cords.

In summary, this study confirms regulatory subunits of phosphatases as promising drug targets. PPP1R15A inhibitors may have applications in the treatment of a broad range of diseases caused by misfolded-protein accumulation.

Sarah Crunkhorn

ORIGINAL RESEARCH PAPER Das, l. et al.

Preventing proteostasis diseases by selective inhibition of a phosphatase regulatory subunit. Science 348, 239-242 (2015)

FURTHER READING Hetz, C. et al. Targeting the unfolded protein response in disease. Nature Rev. Drug Discov. 12, 703-719 (2013)| Tsaytler, H. P. et al. Selective inhibition of a regulatory subunit of protein phosphatase 1 restores proteostasis. Science 332, 91-94 (2011) 